\title{
On solvable subgroups of automorphism groups of right-angled Artin groups
}

\author{
Matthew B. Day
}

October 25, 2009

\begin{abstract}
For any right-angled Artin group, we show that its outer automorphism group contains either a finite-index nilpotent subgroup or a nonabelian free subgroup. This is a weak Tits alternative theorem. We find a criterion on the defining graph that determines which case holds. We also consider some examples of solvable subgroups, including one that is not virtually nilpotent and is embedded in a non-obvious way.
\end{abstract}

\section{Introduction and Background}

\section{$1.1 \quad$ Introduction}

Let $A_{\Gamma}$ be the right-angled Artin group of a finite simplicial graph $\Gamma$ with vertex set $X$, i.e. the group with presentation

$$
\left.A_{\Gamma}=\langle X|\{x y=y x \mid x \text { is adjacent to } y \text { in } \Gamma\}\right\rangle .
$$

In this note we find a combinatorial condition on the graph $\Gamma$ that indicates whether the outer automorphism group Out $A_{\Gamma}$ of $A_{\Gamma}$ contains a nonabelian free group. This extends a result of Gutierrez-Piggott-Ruane [4, Theorem 1.10] which gives a condition for a particular subgroup of Out $A_{\Gamma}$ to be abelian. In fact, our theorem indicates a dichotomy: either Out $A_{\Gamma}$ contains a nonabelian free subgroup or Out $A_{\Gamma}$ is virtually nilpotent. This is a weak Tits alternative theorem. A true Tits alternative theorem would consider all subgroups of Out $A_{\Gamma}$; Charney-Vogtmann [1] recently proved such a theorem for a large class of right-angled Artin groups.

Automorphism groups of right-angled Artin groups are sometimes described as intermediate between automorphism groups of free groups and integer general linear groups, which are extreme examples. This result is 
a first attempt to discern the cases where this idea seems reasonable, since Aut $F_{n}$ and $\operatorname{GL}(n, \mathbb{Z})$ both have nonabelian free subgroups for every $n>1$.

To state the theorem, we review some notions on graphs. Recall that the link $\mathrm{lk}(x)$ of a vertex $x \in \Gamma$ is the set of vertices adjacent to $x$, and the star $\operatorname{st}(x)$ is $\operatorname{lk}(x) \cup\{x\}$. Domination is a useful relation that was considered by Servatius [6, Section IV]:

Definition 1.1. For $x, y \in \Gamma$, say $y$ dominates $x$ if $\operatorname{lk}(x) \subset \operatorname{st}(y)$; denote this by $y \geq x$. Say $x$ and $y$ are domination equivalent if $x \leq y$ and $y \leq x$; denote this by $x \sim y$.

Next we consider the notion of a separating intersection of links defined by Gutierrez-Piggott-Ruane [4, Definition 1.9].

Definition 1.2. The graph $\Gamma$ has a separating intersection of links if there are two vertices $x, y \in \Gamma$ such that (1) $x$ is not adjacent to $y$ and (2) there is a connected component of $\Gamma \backslash(\operatorname{lk}(x) \cap \operatorname{lk}(y))$ not containing $x$ or $y$.

Now we state our main result.

Theorem 1.3. Consider the following conditions on a graph $\Gamma$ :

1. $\Gamma$ contains a domination-equivalent pair of vertices.

2. $\Gamma$ contains a separating intersection of links.

If either condition holds, then Out $A_{\Gamma}$ contains a nonabelian free subgroup. If both conditions fail, then Out $A_{\Gamma}$ is virtually nilpotent.

Proof of Theorem 1.3. If either condition holds, then Out $A_{\Gamma}$ contains a nonabelian free subgroup, by Lemmas 2.1 and 2.2 below. If both conditions fail, then Proposition 2.11 below produces a finite-index nilpotent subgroup.

In Definition 2.10, we define a number $\operatorname{depth}(\Gamma)$ that can be read off of the graph $\Gamma$. If Out $A_{\Gamma}$ is virtually nilpotent, then a certain natural finite-index subgroup of Out $A_{\Gamma}$ turns out to be nilpotent of class $\operatorname{depth}(\Gamma)$. Further, every finite-index nilpotent subgroup of Out $A_{\Gamma}$ has nilpotence class at least $\operatorname{depth}(\Gamma)$. See Proposition 2.11 below for details.

In Section 2.3, we consider a few other conditions that imply Out $A_{\Gamma}$ contains a nonabelian free subgroup. Then we construct examples of graphs $\Gamma$ with Out $A_{\Gamma}$ containing finite-index nilpotent subgroups of arbitrary nilpotence class.

The corollary below follows from Theorem 1.3 by standard arguments. 
Corollary 1.4. If $\Gamma$ is a graph such that Out $A_{\Gamma}$ has a solvable, finite-index subgroup, then every solvable subgroup of Out $A_{\Gamma}$ is virtually nilpotent.

Of course, for $n \geq 3$ the group $\operatorname{GL}(n, \mathbb{Z})$ contains examples of solvable subgroups of infinite index that are not virtually solvable. Given Corollary 1.4. one might conjecture that a solvable subgroup of Out $A_{\Gamma}$ that is not virtually nilpotent must be essentially contained in an embedded copy of $\operatorname{GL}(n, \mathbb{Z})$. In Section 3, we produce an example where this is not the case.

\section{$1.2 \quad$ Background}

We will use the following four classes of automorphisms. The inversion of $x \in X$ is the automorphism sending $x$ to $x^{-1}$ and fixing $X-\{x\}$. If $\pi$ is an automorphism (a symmetry) of the graph $\Gamma$, then the graphic automorphism of $\pi$ is the automorphism sending $x$ to $\pi(x)$ for each $x \in X$. If $x \in X \cup X^{-1}$ and $Y$ is a connected component of $\Gamma-\operatorname{st}(x)$, the partial conjugation of $Y$ by $x$ is the automorphism sending $y$ to $x^{-1} y x$ for each $y \in Y$ and fixing $X-Y$. Denote this automorphism by $c_{x, Y}$. If $x \in X \cup X^{-1}$ and $y \in X$ are distinct and $x \geq y$, then the transvection of $y$ by $x$ is the automorphism sending $y$ to $y x$ and fixing $X-\{y\}$. Denote this automorphism by $\tau_{x, y}$. Sometimes we will refer to the automorphism just defined as the right transvection, and refer to its conjugate by the inversion in $y$ as the left transvection. The multiplier of a transvection $\tau_{x, y}$ is $x$ and the multiplier of a partial conjugation $c_{y, Y}$ is $y$. Servatius defined these automorphisms and showed that they are well defined in [6, Section IV]. Laurence [5] proved the following, which was a conjecture of Servatius.

Theorem 1.5 (Laurence [5]). The finite set of all transvections, partial conjugations, inversions, and graphic automorphisms is a generating set of Aut $A_{\Gamma}$.

Of course the images of these generators form a finite generating set for Out $A_{\Gamma}$. Since we are working in Out $A_{\Gamma}$, in this paper we will demand that partial conjugations are not inner automorphisms. Specifically, whenever we declare that $C_{y, Y}$ is a partial conjugation with multiplier $y$, we also assume $Y$ and $\Gamma \backslash(\operatorname{st}(y) \cup Y)$ are both nonempty.

\subsection{Acknowledgments}

I would like to thank the organizers of the 2009 International Conference on Geometric and Combinatorial Methods in Group Theory and Semigroup Theory, where I started considering the problem in this paper. I am grateful 
to Ruth Charney for commenting on an earlier version of this paper. This research was done under the support of an N.S.F. Mathematical Sciences Postdoctoral Research Fellowship.

\section{Proof of the dichotomy}

\subsection{Conditions for free subgroups}

Lemma 2.1. If $\Gamma$ contains distinct $x, y$ with $x \sim y$, then Out $A_{\Gamma}$ contains a nonabelian free subgroup.

Proof. Let $x, y \in \Gamma$ be vertices with $x \sim y$. Let $G$ be the subgroup of Out $A_{\Gamma}$ generated by the images of $\tau_{x, y}^{2}$ and $\tau_{y, x}^{2}$. The vector space $H_{1}\left(A_{\Gamma} ; \mathbb{R}\right)$ has a basis given by the vertex-set of $\Gamma$; note that $G$ leaves the 2-dimensional subspace $V=\langle[x],[y]\rangle$ invariant. Let $A \subset V$ be the set of $a[x]+b[y]$ with $|a|>|b|$ and let $B \subset V$ be the set of such vectors with $|b|>|a|$. It is easy to see that $A$ and $B$ are nonempty, $\left(\tau_{x, y}^{2}\right)_{*}(B) \subset A$ and $\left(\tau_{y, x}^{2}\right)_{*}(A) \subset B$. Then by the well-known Table-Tennis Lemma (see de La Harpe [3, II.B.24]), we see that $G$ is free of rank 2 .

Lemma 2.2. If $\Gamma$ contains a separating intersection of links, then Out $A_{\Gamma}$ contains a nonabelian free subgroup.

Proof. Let $x, y, z \in \Gamma$ with $y$ not adjacent to $z$ and $\operatorname{lk}(y) \cap \operatorname{lk}(z)$ separating $x$ from both $y$ and $z$. Let $Y$ be the component of $x$ in $\Gamma \backslash \operatorname{lk}(y)$ and let $Z$ be the component of $x$ in $\Gamma \backslash \mathrm{lk} z$. The hypotheses imply that $y \notin Z$ and $z \notin Y$. Let $\widetilde{G}$ be the subgroup of Aut $A_{\Gamma}$ generated by $c_{y, Y}$ and $c_{z, Z}$ and let $G<$ Out $A_{\Gamma}$ be its image. Then $\widetilde{G}$ fixes $y$ and $z$, and therefore contains no nontrivial inner automorphisms. Therefore the projection $\widetilde{G} \rightarrow G$ is an isomorphism.

Map $\widetilde{G}$ to the free group $F_{2}=\langle y, z\rangle$ by sending $\alpha \in \widetilde{G}$ to the unique $w \in\langle y, z\rangle$ with $\alpha(x)=w^{-1} x w$. It is easy to see that this map is a homomorphism with $c_{y, Y}$ mapping to $y$ and $c_{z, Z}$ mapping to $z$. Since an inverse homomorphism $F_{2} \rightarrow \widetilde{G}$ is easy to construct, we see that $\widetilde{G}$ and $G$ are free of rank 2.

\subsection{Conditions for virtual nilpotence}

Lemma 2.3. Suppose $x, y$ and $z$ are in $\Gamma$ such that $x$ is not adjacent to $y$, $\operatorname{lk}(x)$ separates $y$ from $z$ and $\operatorname{lk}(y)$ separates $x$ from $z$. Then $\operatorname{lk}(x) \cap \operatorname{lk}(y)$ separates $x$ and $y$ from $z$ and therefore $\Gamma$ contains a separating intersection of links. 
Proof. Suppose $\operatorname{lk}(x) \cap \operatorname{lk}(y)$ does not separate both $x$ and $y$ from $z$. Then there is a shortest path from $z$ to $x$ or $y$ through $\Gamma \backslash(\operatorname{lk}(x) \cap \operatorname{lk}(y)$. Starting from $z$, the first time this path hits $\operatorname{lk}(x) \cup \operatorname{lk}(y)$ must also be the last, or else there would be a shorter path. Then the hypotheses imply that the point on the path in $\operatorname{lk}(x) \cup \operatorname{lk}(y)$ must also be in $\operatorname{lk}(x) \cap \operatorname{lk}(y)$, a contradiction.

Lemma 2.4. Suppose $x, y \in \Gamma$ with $x$ not adjacent to $y$, we have $x \geq y$ and st $(y)$ separates $\Gamma$. Then $\Gamma$ contains a separating intersection of links.

Proof. Let $z$ be in a component of $\Gamma \backslash \operatorname{st}(y)$ not containing $x$. This means $\mathrm{lk}(y)$ separates $z$ from $x$. Since $x \geq y$, we know $\operatorname{lk}(x) \cap \operatorname{lk}(y)=\operatorname{lk}(y)$, so $\operatorname{lk}(x) \cap \operatorname{lk}(y)$ separates $z$ from $x$ and $y$. Therefore $\Gamma$ contains a separating intersection of links.

Lemma 2.5. Suppose $\Gamma$ does not contain a separating intersection of links. Suppose $\alpha$ and $\beta$ are automorphisms that are either partial conjugations or transvections (or one of each) and that $\alpha$ and $\beta$ fix each other's multipliers. Then $\alpha$ and $\beta$ commute in Aut $A_{\Gamma}$.

Proof. Let $x$ be the multiplier of $\alpha$ and let $y$ be the multiplier of $\beta$. If $x=y^{ \pm 1}$ or $x$ is adjacent to $y$ then $\alpha$ and $\beta$ commute, so assume $x$ and $y$ are distinct and not adjacent. Suppose there is some $z \in \Gamma$ such that neither $\alpha$ nor $\beta$ fixes $z$.

Suppose $x$ is adjacent to $z$. Then $\alpha$ is a transvection (partial conjugations fix the links of their multipliers) and $x \geq z$. If $y \geq z$, then $y$ is adjacent to $x$, counter to our assumption. If $y \nsupseteq z$, then $\beta$ is a partial conjugation and $y$ is not adjacent to $z$. This implies that $x$ and $z$ are in the same connected component of $\Gamma \backslash \operatorname{lk}(y)$, meaning that $\beta$ cannot fix $x$ and change $z$. This contradiction implies that $x$ is not adjacent to $z$, and similarly, that $y$ is not adjacent to $z$.

Suppose $\operatorname{lk}(x)$ does not separate $y$ from $z$. Then $x \geq z$ and $\alpha$ is a partial conjugation. However, in that case $\alpha$ cannot fix $y$ and change $z$. So $\mathrm{lk}(x)$ separates $y$ from $z$, and similarly $\operatorname{lk}(y)$ separates $x$ from $z$. Then by Lemma 2.3, we have that $\Gamma$ does contain a separating intersection of links, which is a contradiction. From this we deduce that for each $z \in \Gamma$, either $\alpha$ fixes $z$ or $\beta$ fixes $z$. This is enough to deduce that $\alpha$ and $\beta$ commute.

In the case that $\alpha$ and $\beta$ are partial conjugations, the following lemma is a special case of Theorem 1.10 from Gutierrez-Piggott-Ruane [4].

Lemma 2.6. Suppose $\Gamma$ does not contain a separating intersection of links. Let $\alpha$ be a partial conjugation. Suppose that $\beta$ is a transvection fixing the 
multiplier of $\alpha$, or that $\beta$ is a partial conjugation (not necessarily fixing the multiplier of $\alpha$ ). Then the images of $\alpha$ and $\beta$ commute in Out $A_{\Gamma}$.

Proof. Suppose the multipliers of $\alpha$ and $\beta$ are distinct (otherwise $\alpha$ and $\beta$ commute). Then possibly by multiplying $\alpha$ and $\beta$ by inner automorphisms, we may assume that $\alpha$ fixes the multiplier of $\beta$, and if $\beta$ is a partial conjugation, we may assume that $\beta$ fixes the multiplier of $\alpha$. The lemma then follows from Lemma 2.5.

Lemma 2.7. Suppose conditions (11) and (2) from Theorem 1.3 both fail. Suppose $\alpha=\tau_{x, y}$ is a transvection and $\beta$ is a transvection or partial conjugation with multiplier $y^{ \pm 1}$. Let $\gamma$ be any commutator of $\alpha$ or $\alpha^{-1}$ with $\beta$ or $\beta^{-1}$. Then $\gamma$ is a transvection or partial conjugation with multiplier $x^{ \pm 1}$ in Out $A_{\Gamma}$. Further, if $\beta$ is a transvection acting on $z^{ \pm 1}$, then so is $\gamma$, and if $\beta$ is a partial conjugation acting on $Y \subset X$, then so is $\gamma$.

Proof. If $\beta$ is a transvection and doesn't fix $x$, then $x \sim y$ and condition (11) holds. If $\beta$ is a partial conjugation, then up to an inner automorphism we may assume that it fixes $x$. So assume $\beta$ fixes $x$.

We claim that $x$ is adjacent to $y$. Let $z \in \Gamma$ be an element not fixed by $\beta$. If $y$ is adjacent to $z$, then $\beta$ is a transvection and $y \geq z$. Since $x \geq y$, this implies that $x \geq z$, and that $x$ is adjacent to $y$. So suppose $y$ is not adjacent to $z$. If $x$ is not adjacent to $y$, then $\operatorname{lk}(y)$ separates $x$ from $z$ since $\beta$ fixes $x$ but not $z$. Then by Lemma 2.4, $\Gamma$ contains a separating intersection of links, contradicting the failure of condition (2). So $x$ is adjacent to $y$. Then the lemma follows by a computation.

Definition 2.8. A domination chain in $\Gamma$ is a sequence of distinct vertices $x_{1}, \ldots, x_{m}$ of $\Gamma$ such that $x_{m} \geq x_{m-1} \geq \cdots \geq x_{1}$. The length of the domination chain $x_{1}, \ldots, x_{m}$ is $m-1$. The domination depth of $x$ is the length of the longest domination chain with $x$ as the dominant member.

Definition 2.9. A domination chain $x_{m} \geq \cdots \geq x_{1}$ is star-separation preserving if $\Gamma \backslash \operatorname{st}\left(x_{1}\right)$ has two components $Y_{1}$ and $Y_{2}$ such that $Y_{i} \not \subset \operatorname{st}\left(x_{m}\right)$ for $i=1,2$. The star-separation depth of $x \in \Gamma$ is

$$
1+\max _{x=x_{m} \geq \cdots \geq x_{1}} \operatorname{length}\left(x_{m} \geq \cdots \geq x_{1}\right)
$$

where the maximum is taken over all star-separation-preserving domination chains. 
Definition 2.10. The depth $\operatorname{depth}(x)$ of a vertex $x \in \Gamma$ is maximum of the domination depth of $x$ and the star-separation depth of $x$. The depth $\operatorname{depth}(\Gamma)$ of $\Gamma$ is the maximum depth of its vertices.

Proposition 2.11. The subgroup $N$ of Out $A_{\Gamma}$ generated by transvections and partial conjugations is finite index in Out $A_{\Gamma}$. If the conditions from Theorem 1.3 both fail, then $N$ is nilpotent of class depth $(\Gamma)$. Further, every finite-index nilpotent subgroup of Out $A_{\Gamma}$ has nilpotence class at least $\operatorname{depth}(\Gamma)$.

Proof. Let $S$ be the finite subset of Out $A_{\Gamma}$ consisting of the identity, the images of transvections (both right and left) and partial conjugations, and their inverses. Let $N$ be the subgroup generated by $S$ and let $P$ be the finite subgroup generated by images of inversions and graphic automorphisms in Out $A_{\Gamma}$. Note that $P$ normalizes $N$ (since conjugation by $P$ leaves $S$ invariant). By Laurence's theorem (Theorem 1.5), Out $A_{\Gamma}=P N$ and therefore $N \triangleleft$ Out $A_{\Gamma}$. By a classical group isomorphism theorem, Out $A_{\Gamma} / N \cong P /(P \cap N)$ and therefore $N$ is finite-index in Out $A_{\Gamma}$. (In fact, the failure of condition (1) implies that $N \cap P=1$ and Out $A_{\Gamma} \cong P \ltimes N$, as can be seen from the presentation for Aut $A_{\Gamma}$ in Day [2, Theorem 2.7].)

Let $k=\operatorname{depth}(\Gamma)$. Let $S_{0}=\{1\}$, and let $S_{i}$ be the union of $\{1\}$ with the set of tranvsections $\tau_{x, y}$ with $\operatorname{depth}(x)-\operatorname{depth}(y) \geq k-i+1$ (and left transvections satisfying the same condition) and partial conjugations $c_{y, Y}$ with $\operatorname{depth}(y) \geq k-i+1$ for $i=1, \ldots, k$. The $S_{i}$ are nested and $S$ is $S_{k}$.

Let $\alpha \in S_{i}$ and $\beta \in S_{j}$, for $1 \leq i, j \leq k$. By Lemmas 2.5, 2.6] and 2.7, we see that if $[\alpha, \beta]$ is nontrivial, then $i+j>k+1$ and $[\alpha, \beta]$ is a member of $S_{i+j-k-1}$. Since $i, j \leq k$, we have that $i+j-k-1<i, j$. This is enough to deduce that $N$ is nilpotent of class at most $k$.

Select $x_{k} \in \Gamma$ with $\operatorname{depth}\left(x_{k}\right)=k$. By definition, there is a domination chain $x_{k} \geq \cdots \geq x_{1}$ in $\Gamma$, such that either $x_{1}$ dominates a vertex $x_{0}$, or $\Gamma \backslash \operatorname{st}\left(x_{1}\right)$ has two components $Y_{1}, Y_{2}$ with $Y_{i} \not \subset$ st $\left(x_{k}\right)$ for $i=1,2$ (depending on whether $\operatorname{depth}\left(x_{k}\right)$ is the domination depth or the star-separation depth, respectively). In the first of these cases, let $\alpha_{1}$ denote the transvection $\tau_{x_{1}, x_{0}}$, and in the second of these cases, let $\alpha_{1}$ denote the partial conjugation $c_{x_{1}, Y_{1}}$. For $i=2, \ldots, k$, let $\alpha_{i}$ be the transvection $\tau_{x_{i}, x_{i-1}}$. Then by Lemma 2.7 , the element

$$
\left[\cdots\left[\left[\alpha_{1}, \alpha_{2}\right], \alpha_{3}\right], \ldots, \alpha_{k}\right] \in \operatorname{Out} A_{\Gamma}
$$

is either a transvection $\tau_{y, x_{0}}$ or a partial conjugation $c_{y, Y}$, where $y=x_{k}^{ \pm 1}$ and $Y=Y_{1} \backslash \operatorname{st}(y)$. If it is $\tau_{y, x_{0}}$, it is obviously nontrivial in Out $A_{\Gamma}$. If it is $c_{y, Y}$, it is nontrivial in Out $A_{\Gamma}$ since there is an element of $Y_{1} \backslash \operatorname{st}(y)$ that 
is conjugated and an element of $Y_{2} \backslash \operatorname{st}(y)$ that is not conjugated. So the nilpotence class of $N$ equals $\operatorname{depth}(\Gamma)$.

Now suppose that $N^{\prime \prime}$ is a nilpotent, finite-index subgroup of Out $A_{\Gamma}$. Then $N^{\prime \prime}$ intersects $N$ in a finite index subgroup $N^{\prime}$. Each of the $\alpha_{1}, \ldots, \alpha_{k}$ from the previous paragraph is of infinite order. Since $N^{\prime}$ is finite index in $N$, the intersection $N^{\prime} \cap\left\langle\alpha_{i}\right\rangle$ is finite index in $\left\langle\alpha_{i}\right\rangle$ for each $i$. In particular, each $N^{\prime} \cap\left\langle\alpha_{i}\right\rangle$ is nontrivial. So we have $a_{1}, \ldots, a_{k} \in \mathbb{Z}$ with $\alpha_{i}^{a_{i}} \in N^{\prime}$ for each $i$. Then by the same reasoning as in the previous paragraph, we see that

$$
\left[\cdots\left[\left[\alpha_{1}^{a_{1}}, \alpha_{2}^{a_{2}}\right], \alpha_{3}^{a_{3}}\right], \ldots, \alpha_{k}^{a_{k}}\right] .
$$

is nontrivial. From this, we see the nilpotence class of $N^{\prime}$ is also $\operatorname{depth}(\Gamma)$, and the nilpotence class of $N^{\prime \prime}$ is at least $\operatorname{depth}(\Gamma)$.

The following needs no further proof.

Corollary 2.12. The group Out $A_{\Gamma}$ is virtually abelian if and only if both conditions from Theorem 1.3 fail and $\operatorname{depth}(\Gamma) \leq 1$.

Remark 2.13. It has long been known that Out $A_{\Gamma}$ is finite if and only if $\Gamma$ contains no pair of vertices $x, y$ with $x \geq y$ and $\Gamma$ contains no vertex $x$ with $\Gamma-\operatorname{st}(x)$ disconnected. This is an easy corollary of Theorem 1.5 .

\section{$2.3 \quad$ Examples}

Corollary 2.14. The group Out $A_{\Gamma}$ has a nonabelian free subgroup if any of the following conditions on $\Gamma$ hold:

- $\Gamma$ is disconnected.

- $\Gamma$ contains a cut-vertex that breaks $\Gamma$ into three or more components.

- $\Gamma$ contains non-adjacent vertices $x$ and $y$ with $x \geq y$ and $\operatorname{st}(y)$ separating $\Gamma$.

- $\Gamma$ contains pairwise non-adjacent vertices $x, y$ and $z$ with $x \geq y \geq z$.

Proof. In each case we find a domination-equivalent pair of vertices or a separating intersection of links in $\Gamma$, and Theorem 1.3 implies the corollary. The final condition is a special case of the second to last condition, which implies $\Gamma$ has a separating intersection of links by Lemma 2.4 .

Now suppose that $\Gamma$ is disconnected. If $\Gamma$ is edgeless, then any two vertices are domination equivalent. Otherwise some component of $\Gamma$ has at 
least two vertices. If each component of $\Gamma$ is a complete graph, then any two vertices in the same component are domination equivalent. So we have some component of $\Gamma$ that contains two nonadjacent vertices. Then $\Gamma$ contains a separating intersection of links (for $x$ and $y$ not adjacent, $\operatorname{lk}(x) \cap \operatorname{lk}(y)$ separates $x$ and $y$ from any vertex in another component).

Now suppose $\Gamma$ contains a cut-vertex $z$ that breaks $\Gamma$ into at least three components. Without loss of generality we assume $\Gamma$ is connected. If the valence of $z$ is less than 2 , then $\Gamma \backslash\{z\}$ has only one component. If for for each pair of distinct $x, y \in \operatorname{lk}(z)$, either $x$ is adjacent to $y$ or $\operatorname{lk}(x) \cap \operatorname{lk}(y)$ contains two or more elements, then $\Gamma \backslash\{z\}$ has only one component. Therefore $\Gamma$ contains distinct, non-adjacent vertices $x$ and $y$ with $\operatorname{lk}(x) \cap \operatorname{lk}(y)=\{z\}$. Then $\Gamma \backslash(\operatorname{lk}(x) \cap \operatorname{lk}(y))$ has at least three components and $\Gamma$ has a separating intersection of links.

Proposition 2.15. For each $k \geq 0$, there is a graph $\Gamma_{k}$ such that Out $A_{\Gamma_{k}}$ contains a finite-index subgroup of nilpotence class $k$.

Proof. For each $k$, we will construct a graph $\Gamma_{k}$ with $\operatorname{depth}\left(\Gamma_{k}\right)=k$ and such that $\Gamma_{k}$ satisfies the hypotheses of Proposition 2.11. We can take $\Gamma_{0}$ to be the graph with one vertex.

Now fix $k>0$. For the vertex set of $\Gamma_{k}$, we will take a set of $2 k+2$ vertices labeled as $x_{0}, \ldots, x_{k}, y_{0}, \ldots, y_{k}$. Take the induced subgraph on $\left\{x_{i}\right\}_{i}$ to be the complete graph on $k$ vertices, and similarly for $\left\{y_{i}\right\}_{i}$. Further, connect $x_{i}$ to $y_{j}$ by an edge if $i+j>k$. These are the only edges of $\Gamma_{k}$.

Then $x_{k} \geq y_{0}, y_{k} \geq x_{0}$, and for $0 \leq i<j \leq k$ we have $x_{j} \geq x_{i}$ and $y_{j} \geq y_{i}$. Since $k>0$, these are the only pairs which satisfy the domination relation. In particular, there are no domination-equivalent pairs. There are no vertices whose stars separate $\Gamma$, so the star-separation depth of all vertices is trivial. We compute all depths as equal to domination depths, and find $\operatorname{depth}\left(x_{i}\right)=\operatorname{depth}\left(y_{i}\right)=i$ for $i=0, \ldots, k$. Therefore $\operatorname{depth}\left(\Gamma_{k}\right)=k$.

The only non-adjacent pairs of vertices are $\left(x_{i}, y_{j}\right)$ and $\left(x_{j}, y_{i}\right)$ for $i+j \leq$ $k$. For such $i, j$, every element of $\Gamma_{k}$ is adjacent to either $x_{i}$ or $y_{j}$. In particular, every element of $\Gamma_{k} \backslash\left(\operatorname{lk}\left(x_{i}\right) \cap \operatorname{lk}\left(y_{j}\right)\right)$ has a path of length one to either $x_{i}$ or $y_{j}$ (and similarly for $x_{j}$ and $y_{i}$ ). Therefore $\Gamma_{k}$ does not contain a separating intersection of links.

\section{A non-nilpotent solvable subgroup}

Whenever $Y \subset X$ is a clique with $x \sim y$ for all $x, y \in Y$, the transvections of elements of $Y$ acting on each other generate an embedded copy of $\mathrm{SL}(|Y|, \mathbb{Z})$ 
inside Out $A_{\Gamma}$. When we have such a copy of $\mathrm{SL}(n, \mathbb{Z})$, say it is canonically embedded. Of course one can find non-virtually-nilpotent solvable subgroups of Out $A_{\Gamma}$ inside canonically embedded copies of $\operatorname{SL}(n, \mathbb{Z})$ for $n \geq 3$. Given Corollary 1.4, one might conjecture that when $G<$ Out $A_{\Gamma}$ is solvable but not virtually nilpotent, there is $H<$ Out $A_{\Gamma}$ a canonically embedded copy of $\mathrm{SL}(n, \mathbb{Z})$, such that $H \cap G$ is not virtually nilpotent. However the following example is not of this type.

Proposition 3.1. Let $\Gamma$ be the graph on three vertices $\{a, b, c\}$ with a single edge from a to $b$. Let $G$ be the subgroup of Out $A_{\Gamma}$ generated by the images of the elements $\left\{\tau_{a, c}, \tau_{b, c}, \tau_{a, b} \tau_{b, a}\right\}$. Then $G$ is a solvable group and is not virtually nilpotent.

The intersection of $G$ with the unique canonically embedded copy of $\mathrm{SL}(n, \mathbb{Z})$ in Out $A_{\Gamma}$ is not virtually nilpotent.

Proof. It is apparent that $G$ does not contain any inner automorphisms, so $G$ is isomorphic to the subgroup of Aut $A_{\Gamma}$ generated by these generators. Let $\alpha=\tau_{a, c}, \beta=\tau_{b, c}$, and let $\gamma=\tau_{a, b} \tau_{b, a}$. Since $a$ commutes with $b$, we know that $\alpha$ commutes with $\beta$. A computation shows that $\gamma \alpha \gamma^{-1}=\alpha^{2} \beta$ and $\gamma \beta \gamma^{-1}=\alpha \beta$. It is easy to see that $\langle\alpha, \beta\rangle \cap\langle\gamma\rangle=1$. From this we can see that $G$ is the semidirect product $\mathbb{Z} \ltimes \mathbb{Z}^{2}$, where $\mathbb{Z}$ acts on $\mathbb{Z}^{2}$ by the matrix $\left(\begin{array}{ll}2 & 1 \\ 1 & 1\end{array}\right)$. So $G$ is solvable.

On the other hand, for all $k>0$, the centralizer of $\gamma^{k}$ in $G$ is $\langle\gamma\rangle$. Let $H$ be a finite index subgroup of $G$. Then $H$ contains a positive power of $\gamma$ and an element of $G$ outside of $\langle\gamma\rangle$. So $H$ has trivial center and is therefore not nilpotent. In fact $G$ is isomorphic to a lattice in the 3-dimensional Lie group sol; see Thurston [7, Example 3.8.9] for explanation.

The only canonically embedded copy of any $\mathrm{SL}(n, \mathbb{Z})$ in Out $A_{\Gamma}$ is generated by $\tau_{a, b}$ and $\tau_{b, a}$. However, the intersection of $G$ with this subgroup is a copy of $\mathbb{Z}$.

\section{References}

[1] Ruth Charney and Karen Vogtmann, Subgroups and quotient groups of automorphism groups of RAAGs, ArXiv preprint, arXiv:0909.2444, 2009.

[2] Matthew B. Day, Peak reduction and finite presentations for automorphism groups of right-angled Artin groups, Geom. Topol. 13 (2009), no. 2, 817-855.

[3] Pierre de la Harpe, Topics in geometric group theory, Chicago Lectures in Mathematics, University of Chicago Press, Chicago, IL, 2000. 
[4] Mauricio Gutierrez, Adam Piggott, and Kim Ruane, On the automorphisms of a graph product of abelian groups, ArXiv preprint, arXiv:0710.2573, 2007.

[5] Michael R. Laurence, A generating set for the automorphism group of a graph group, J. London Math. Soc. (2) 52 (1995), no. 2, 318-334.

[6] Herman Servatius, Automorphisms of graph groups, J. Algebra 126 (1989), no. 1, 34-60.

[7] William P. Thurston, Three-dimensional geometry and topology. Vol. 1, Princeton Mathematical Series, vol. 35, Princeton University Press, Princeton, NJ, 1997, Edited by Silvio Levy.

Department of Mathematics 253-37

California Institute of Technology

Pasadena, CA 91125

E-mail: mattday@caltech.edu 